\title{
Lupus miliaris disseminatus faciei: A report with a new dermoscopy finding
}

\section{Basavapura Madegowda Shashi Kumar', Allaranda Somiiah Savitha ${ }^{2}$}

${ }^{1}$ Consultant Dermatologists, Sparsha Skin Care Clinic, Mandya, Karnataka, India, ${ }^{2}$ Associate Professor, Sapthagiri Institute of Medical Sciences and Research Centre Bangalore, Karnataka, India

Corresponding author: Dr. Basavapura Madegowda Shashi Kumar, E-mail: shashikumarbm@gmail.com

\begin{abstract}
Lupus miliaris disseminatus faciei (LMDF) is a rare inflammatory disorder characterized by asymptomatic papular lesions mainly over the central part of the face, common in young adults and with a spontaneously resolving course. Its exact etiology remains unknown, its treatment is problematic, and there is a lack of controlled studies on LMDF. Histopathology shows dermal epithelioid granulomas with central necrosis and surrounding lymphocytic infiltrate with multinucleate giant cells. Dermoscopic findings published show discrete focal orangish structureless areas located around follicular openings with whitish keratotic plugs. Herein, we report a case of LMDF with dermoscopy showing stellate scar signs and structureless white areas.
\end{abstract}

Key words: Lupus miliaris; Dermoscopy; Acne agminata

\section{INTRODUCTION}

Lupus miliaris disseminatus faciei (LMDF), also known as acne agminata, is a rare chronic granulomatous disorder of unknown etiology first described by Fox in 1878 [1]. Its clinical features are asymptomatic bilaterally symmetric papules characteristically distributed over the central part of the face [2]. Histopathology shows epithelioid cell granulomas with central necrosis. Dermoscopic findings include discrete focal orangish structureless areas located around follicular openings with whitish keratotic plugs [3]. Although its classical clinical features and histopathology have been described, reports on the dermoscopic findings are scarce. Herein, we report a new case of LMDF with varied dermoscopic features not described before.

\section{CASE REPORT}

A previously healthy 21 -year-old male consulted us about asymptomatic lesions over the face persistent for three months prior. There were no systemic complaints or concomitant systemic disorders. Upon examination, discrete erythematous papules were seen on the face. Several lesions had a pus top. The lesions were distributed symmetrically, more over the central part of the face, namely the nose, cheeks, perioral areas, and chin (Fig. 1). There were no telangiectasias. Other parts of the body-the mucosa, palms, soles-were uninvolved. The patient denied taking oral or topical medication prior to consultation.

Histopathology of the papule on the chin revealed a single large focus of tuberculoid granuloma involving the middle and deep layer of the reticular dermis, consisting of lymphocytes, epithelioid cells, Langhans giant cells, foreign-body giant cells, and plasma cells. The center of the granuloma showed a large focus of fibrinoid necrosis. The periphery showed abundant fibroplasia. The rest of the dermis showed moderately dense perivascular and periappendegeal inflammatory infiltrate (Figs. 2a and 2b). Staining for microorganisms with the periodic acid-Schiff stain, Grocott stain, and Ziehl-Neelsen stain was negative. A routine hemogram and biochemical investigations,

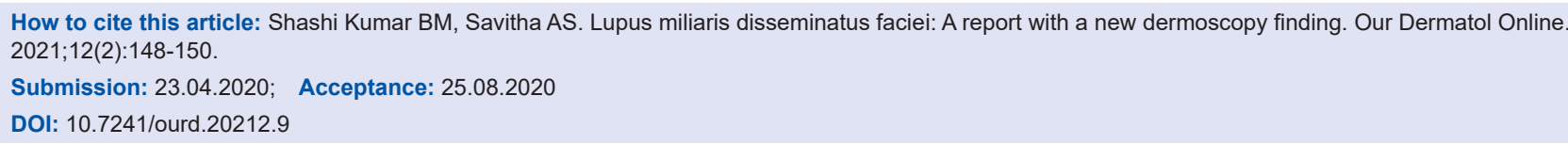




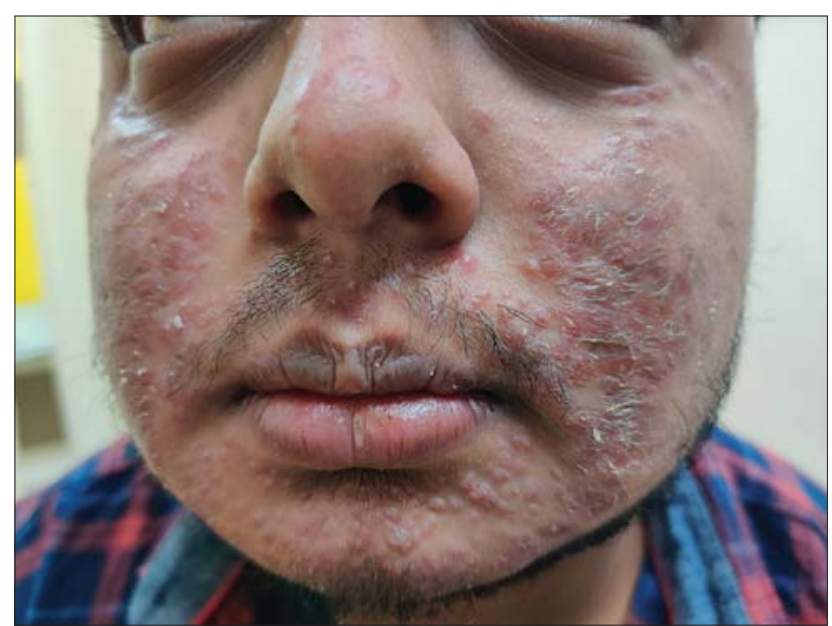

Figure 1: Erythematous papules on the central face, with scaling, several lesions, and a pus top.

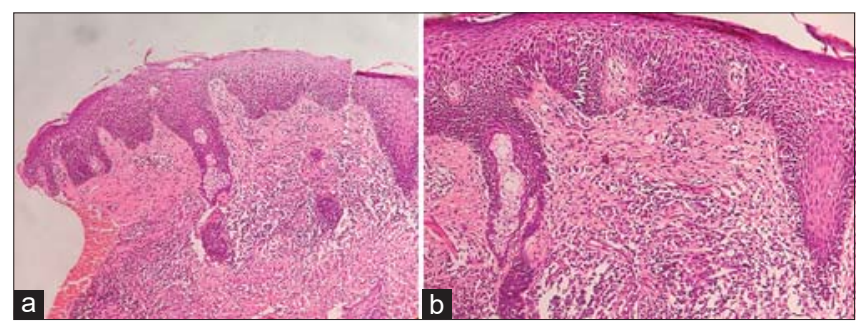

Figure 2: (a) A single large focus of tuberculoid granuloma involving the middle and deep layer of the reticular dermis, with the periphery showing abundant fibroplasia, and the rest of the dermis with moderately dense perivascular and periappendegeal inflammatory infiltrate; and an unremarkable epidermis (H\&E, 10x). (b) A tuberculoid granuloma consisting of lymphocytes, epithelioid cells, Langhans giant cells, foreign-body giant cells, and plasma cells with a center demonstrating a large focus of fibrinoid necrosis (H\&E, 40x).

including serum calcium and angiotensin-converting enzyme, were normal, and the Mantoux test was negative. A chest X-ray was normal. Basing on the clinical signs and histopathology, a diagnosis of LMDF was reached.

Dermoscopy showed white areas of the stellar scar sign, structureless white areas, follicular plugs, and brownish-red globules (Fig. 3). The patient was started on dapsone $100 \mathrm{mg}$ OD. Lesions gradually decreased in number and size over a period of 4 months. The patient is still under observation for recurrence.

\section{DISCUSSION}

Lupus miliaris disseminatus faciei (LMDF) is an uncommon but very distinct chronic inflammatory dermatosis characterized by reddish-yellow or yellowishbrown papules on the central face, particularly on and around the eyelids [4]. The papules may occur alone

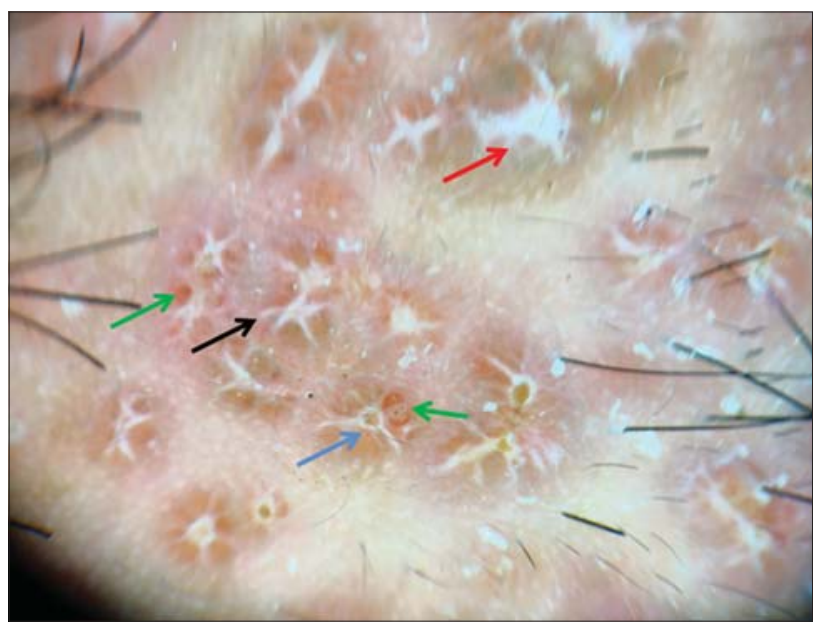

Figure 3: Dermoscopy of the facial lesion showing white areas of the stellate scar sign (black arrow), white structureless areas (red arrow), keratotic follicular plugs (blue arrow), and brownish-red globules (green arrow).

or in crops, may be follicular or non-follicular, and may sometimes have pustular tops [2].

Extrafacial lesions in the axillae, scalp, trunk, genitalia, neck, hands, and legs have been described [5]. LMDF usually affects young adults of both sexes between the second and fourth decade of life. Its exact etiopathogenesis remains unclear. Various hypotheses have believed it to be a form of cutaneous tuberculosis, tuberculid, sarcoidosis, and granulomatous rosacea, but research failed to support these associations [6]. LMDF is distinguished from granulomatous rosacea by the lack of erythema and telangiectasia and by the presence of caseating necrosis in the granulomas [5]. The disease heals spontaneously often over a chronic course of months to years, but treatment is required to prevent scarring. However, recurrences are not uncommon and may lead to more scarring [7].

According to Errichetti et al., the most characteristic dermatoscopic feature of LMDF is discrete (nonconfluent) focal orangish structureless areas located around follicular openings [3]. Such a pattern is due to the peculiar histological background of this kind of dermatosis, with perifollicular granulomatous infiltrate along with follicular hyperkeratosis. Ayhan et al. also described linear and hairpin vessels, targetoid flame-like follicular plugs, and ellipsoid keratotic follicular plugs upon dermoscopy [8]. Our case had white areas of the stellar scar sign, structureless white areas, keratotic follicular plugs, and brownish-red globules, previously unreported.

Due to the lack of a controlled trial standard, there is no recommended treatment for LMDF. Topical steroids 
are ineffective. Several reports have been published on variably successful treatment with intralesional or systemic corticosteroids, topical tacrolimus, tetracyclines, metronidazole, erythromycin, dapsone, anti-tuberculous antibiotics, isotretinoin, chloroquine, and clofazimine. There have also been reports on successful laser therapy of lesions and scars with a 1,565 $\mathrm{nm}$ nonablative fractionated laser, a $1,450 \mathrm{~nm}$ diode laser, and a carbon dioxide laser [6].

\section{CONCLUSION}

Dermatoscopic findings help to aid the diagnosis of LMDF and differentiate it from other granulomatous conditions. Since LMDF is relatively uncommon, more dermatoscopic studies are required to establish its classical dermoscopic features.

\section{Consent}

The examination of the patient was conducted according to the principles of the Declaration of Helsinki.

The authors certify that they have obtained all appropriate patient consent forms, in which the patients gave their consent for images and other clinical information to be included in the journal. The patients understand that their names and initials will not be published and due effort will be made to conceal their identity, but that anonymity cannot be guaranteed.

\section{REFERENCES}

1. Fox T. Clinical Lecture on disseminated follicular lupus (simulating acne). Lancet. 1878;112:75-6.

2. Esteves T, Faria A, Alves R, Marote J, Viana I, Vale E. Lupus miliaris disseminatus faciei: a case report. Dermatol Online J. 2010;16:10.

3. Errichetti E, Stinco G. Dermatoscopy of granulomatous disorders. Dermatol Clin. 2018;36:369-75.

4. Al-Mutairi N. Nosology and therapeutic options for lupus miliaris disseminatus faciei. J Dermatol. 2011;38:864-73.

5. van de Scheur MR, van der Waal RIF, Starink TM. Lupus miliaris disseminatus faciei: a distinctive rosacea-like syndrome and not a granulomatous form of rosacea. Dermatology. 2003;206:120-3.

6. Schaarschmidt ML, Schlich M, Staub J, Schmieder A, Goerdt S, Peitsch WK. Lupus miliaris disseminatus faciei: not only a facial dermatosis. Acta Derm Venereol. 2017;97:655-6.

7. Zonunsanga, Khare AK, Manisha, Saini P. Recurrent lupus miliaris disseminatus faciei: a case report. Our Dermatol Online. 2015;6:43-5.

8. Ayhan E, Alabalik U, Avci Y. Dermoscopic evaluation of two patients with lupus miliaris disseminates faciei. Clin Exp Dermatol. 2014;39:500-2.

Copyright by Basavapura Madegowda Shashi Kumar, et al. This is an open-access article distributed under the terms of the Creative Commons Attribution License, which permits unrestricted use, distribution, and reproduction in any medium, provided the original author and source are credited.

Source of Support: Nil, Conflict of Interest: None declared. 